\title{
Prospects for radio observations of Particle-Accelerating Colliding-Wind Binaries with the Giant Metrewave Radio Telescope
}

\author{
Michaël De Becker ${ }^{1 *}$, C. H. Ishwara-Chandra ${ }^{2}$, Paula Benaglia ${ }^{3,4}$ \\ ${ }^{1}$ Space sciences, Technologies and Astrophysics Research (STAR) Institute, University of Liège, \\ Quartier Agora, 19c, Allée du 6 Août, B5c, B-4000 Sart Tilman, Belgium \\ ${ }^{2}$ National Centre for Radio Astrophysics, TIFR, Post Bag No. 3, Ganeshkhind Post, \\ 411007 Pune, India \\ ${ }^{3}$ Instituto Argentino de Radioastronomía (CONICET;CICPBA), C.C. No 5, 1894, \\ Villa Elisa, Argentina \\ ${ }^{4}$ Facultad de Ciencias Astronómicas y Geofísicas, UNLP, Paseo del Bosque s/n, 1900, \\ La Plata, Argentina
}

\begin{abstract}
Synchrotron radiation identified in the radio domain for several tens of binary systems made of massive stars provides compelling evidence that a particle acceleration process is at work in these objects, hence their Particle-Accelerating Colliding-Wind Binaries (PACWB) status. Measurements of the synchrotron radio emission allow to investigate the non-thermal physics and to derive some of their properties. Non-thermal spectra are known to increase at lower frequencies, while thermal ones increase toward higher frequencies. In this context, it is worth investigating the expectations from longer wavelengths such as those measured by the Giant Metrewave Radio Telescope in India, in complementarity with the most abundant measurements at centimetric wavelengths obtained with other radio observatories such as the VLA or ATCA.
\end{abstract}

Keywords: Massive stars - Binaries - Radio waves - Acceleration of particles - Non-thermal processes

\section{A few words on PACWBs}

One of the main features characterizing massive stars is their strong stellar winds. These winds are produced by the strong radiation pressure driven by the intense radiation field of massive stars (e.g. Puls et al. 2008 for a review). The mass loss rate of these stars can be in the range of $10^{-6}$ $10^{-5} \mathrm{M}_{\odot} \mathrm{yr}^{-1}$, and the asymptotic velocity they can reach (i.e. the so-called terminal velocity) is often of the order of 2000-3000 $\mathrm{km} \mathrm{s}^{-1}$. In massive binaries - or higher multiplicity systems - stellar winds collide and produce strong hydrodynamic shocks, as illustrated in Fig. 1. As a consequence, such systems constitute highly valuable laboratories to study shock physics in astrophysical sources.

*Michael.DeBecker@uliege.be 


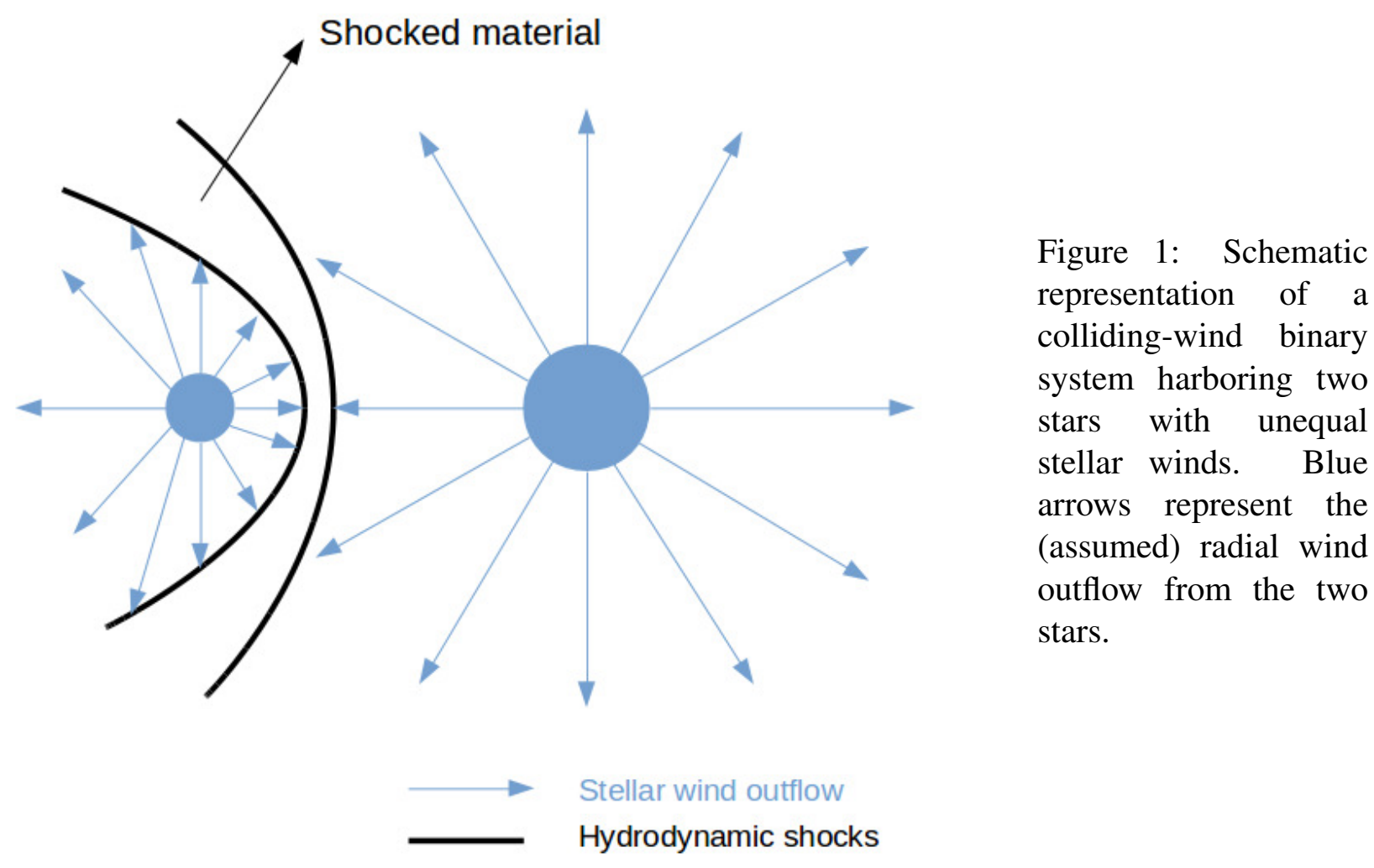

It is important to clarify from the beginning that the present discussion is relevant to the case of O-type stars, early B-type stars, and their evolved counter parts referred to as Wolf-Rayet stars. The fundamental reason is that the shock physics one is considering here requires to be significantly fed with mechanical energy. This puts stringent constraints on the wind parameters (mass loss rate and terminal velocity) which are required to drive to a sufficient level emission processes such as described in Sect. 2. Later-type stars wouldn't be able to significantly contribute as their winds are characterized by a significantly lower kinetic power, because of lower mass loss rates and wind velocities.

Among the physical processes related to shock physics, particle acceleration is of high interest. The most likely mechanism responsible for the acceleration of charged particles up to relativistic velocities in the presence of shocks is the Diffusive Shock Acceleration (DSA) process (e.g. Drury 1989). This acceleration process is considered to be the source of Galactic cosmic-rays produced by supernova remnants, including relativistic electrons responsible for their strong synchrotron radio emission (Dubner \& Giacani 2015). The DSA process is also believed to be the main reason for the presence of relativistic electrons in shocks in clusters of galaxies, at the origin of their diffuse radio emission (Ensslin et al. 1998). The sub-set of colliding-wind binaries that are known to accelerate particles are called Particle-Accelerating Colliding-Wind Binaries (PACWBs). PACWBs can thus, to some extent, be seen as a scaled down version of shocks due to cluster collision, but the underlying physics remains the same. Such an acceleration process is believed to be responsible for the acceleration of electrons, protons, helium nuclei and even heavier nuclei. The existence of this population of relativistic particles opens the door for non-thermal emission processes. In the specific context of relativistic electrons, in the radio domain, the only active non-thermal continuum emission process is synchrotron radiation. Details on synchrotron emission in the context of PACWBs are developed in Sect. 2.

Most PACWBs are identified as particle accelerators thanks to the detection of synchrotron emission in their radio spectrum. To date about 40 PACWBs are included in the catalogue (De Becker \& 
Raucq 2013), and prospects are explored to identify additional members (De Becker et al. 2017). A general discussion on this class of objects can be found in De Becker (2018a, Proceedings of the 1st BINA workshop).

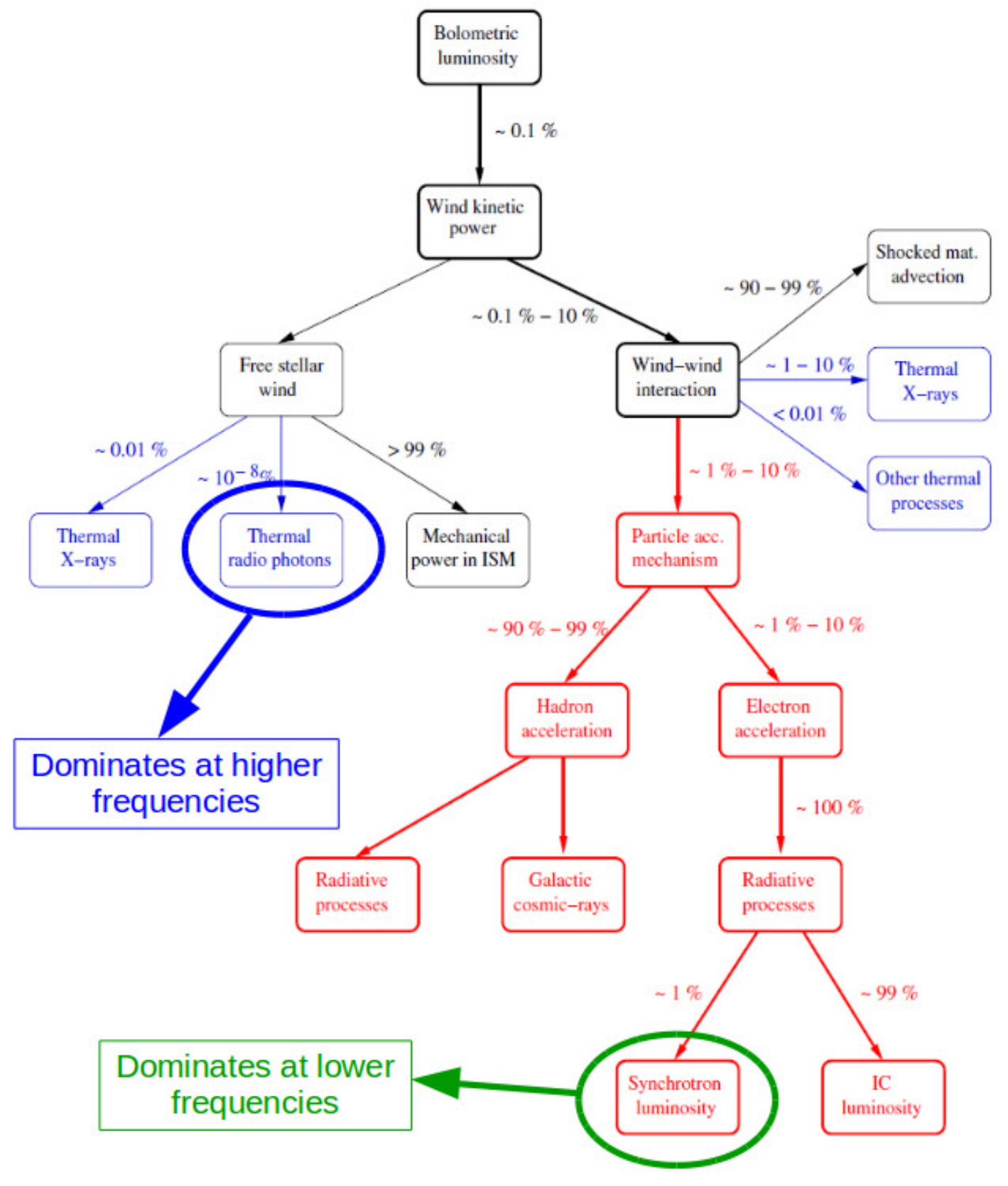

Figure 2: Energy buget of PACWBs. Modified version of a figure reproduced from De Becker \& Raucq (2013) with permission (C) ESO).

It is always instructive to address the issue of energy budget in astrophysical objects, in order to figure out where the energy that feeds some specific physical processes is coming from. A schematic representation of the energy budget in PACWBs is illustrated in Fig. 2. The starting point of the discussion is the bolometric luminosity of the stars in the system. This is the pool of energy that will drive the stellar wind through a conversion of the radiative energy into mechanical energy. In term of energy conversion per unit time, one may speak about wind kinetic power determined by the wind mass loss rate and its terminal velocity. Only a fraction of total wind kinetic power is actually 
injected into the wind-wind interaction region, whereas the remaining part of the wind is not colliding and disperses into the interstellar medium. The amount of energy injected in the colliding-wind region constitutes the reservoir that will feed shock physics. Among other aspects, in the framework of the investigation of non-thermal processes (red part of Fig. 2), let's focus on the energy that is injected into particle acceleration. That energy will be split into electrons and hadrons. The energy that will be injected into synchrotron emission is taken from relativistic electrons. One clearly sees that the synchrotron emission process appears downstream of a series of energy conversion processes, and one can anticipate that only a tiny fraction of the initial energy is likely to be radiated into non-thermal radio emission. Depending on the system, only a fraction of $10^{-12}$ up to $10^{-8}$ of the bolometric luminosity of the stars may be converted into synchrotron radio emission (depending notably on the wind properties, the particle acceleration efficiency, or the geometry of the colliding wind region). The relatively weak emission level will be of high importance when considering instruments characterized by the required sensitivity.

Beside that, radio emission won't be made only of synchrotron emission. A quick look at the blue part of Fig. 2 shows that the stellar winds of massive stars, apart from any collision, are thermal radio emitters (with a fraction of the bolometric luminosity of the order of $10^{-11}-10^{-10}$ converted into thermal radio emission). One can thus anticipate that PACWBs will be composite radio emitters, with a thermal contribution coming from the stellar winds $\left(S_{\nu} \propto \nu^{0.6}\right.$ : Wright \& Barlow 1975, Panagia $\&$ Felli 1975), and a non-thermal contribution coming from the wind-wind interaction region $\left(S_{\nu} \propto\right.$ $\nu^{-\alpha}$, with $\alpha$ being a spectral index set as positive in the conventions adopted here: Blumenthal \& Gould 1970).

Considering the opposite trends displayed by the frequency dependences of these two emission processes, one can immediately notice that thermal emission will dominate at higher frequencies and synchrotron emission will dominate at lower frequencies, as emphasized in Fig. 3.

\section{Synchrotron emission from PACWBs}

As stated above, synchrotron emission is to date the main tracer of particle acceleration which allows to identify PACWBs among colliding-wind binaries. Typically, synchrotron emission is revealed through a negative slope of the $\log S_{\nu}$ vs $\log \nu$ spectrum and/or a high brightness temperature (typically above $10^{6} \mathrm{~K}$, significantly larger than that of the thermal wind at a few $\sim 10^{4} \mathrm{~K}$ ). In addition, as one is dealing with binary systems, the synchrotron emission is very likely to vary as a function of the orbital phase. The reason is twofold: (i) in eccentric orbits the stellar separation is changing and it causes significant changes in the physical conditions in the wind-wind interaction region, (ii) synchrotron photons are likely to be significantly absorbed by the stellar wind material, and the absorbing column along the line of sight can change drastically (strong orientation effect). The measurement of a variable radio emission constitutes therefore another precious indicator of non-thermal radio emission in massive binaries.

Most radio measurements of PACWBs so far have been performed typically in the $1-10 \mathrm{GHz}$ range, using facilities such as the Very Large Array (VLA) or the Australia Telescope Compact Aray (ATCA). Among other studies, let's mention the works by Abbott et al.(1984, 1986), Benaglia et al. (2005, 2006), Blomme et al. (2007), Blomme \& Volpi (2014), Dougherty et al. (2005), Montes et al. (2009), Setia Gunawan et al. (2000, 2003), Williams et al. (1990, 1994), and many others.

The composite nature of the radio emission from PACWBs is illustrated by the schematic spectrum shown in Fig. 3. The contamination of the radio spectrum by the thermal emission depends intimately on the frequency. The amplitude of this contamination depends on the system. At centimetric frequencies where most systems have been observed so far, the thermal emission component 


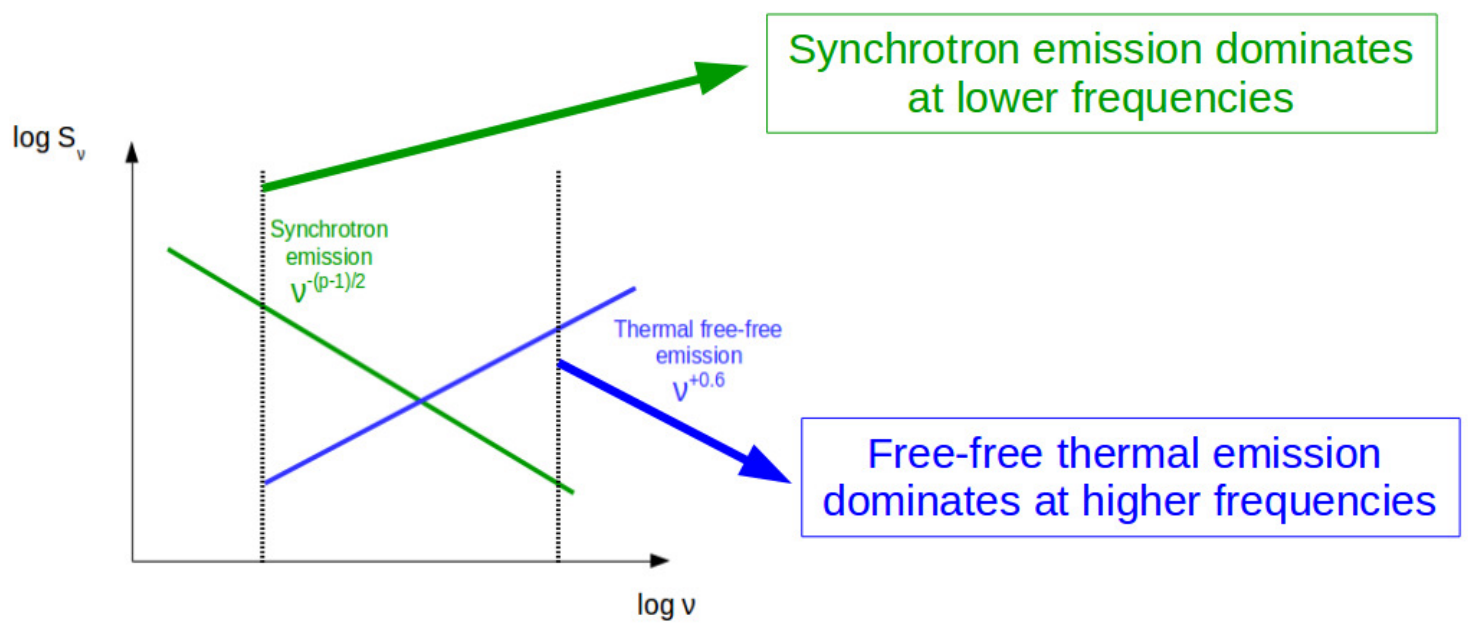

Figure 3: Non-thermal (synchrotron) and thermal (free-free) emission components contributing to the radio spectrum of PACWBs. The vertical lines stand for any arbitrary low and higher frequencies where the ratio of both contributions are very different, with consequences on the measurability of the synchrotron component.

can be in the range between a few percent to a substantial fraction of the total measured radio emission (affected by free-free absorption of the synchrotron emission component). Lower frequencies allow us to minimize thermal contamination (below a few percent) and to maximize the synchrotron emission level, which is ideal to investigate the non-thermal physics of PACWBs (though free-free absorption may provide other limitations to the observations, see Sect. 3). This points a priori to a very good adequacy of lower frequency measurements using the upgraded Giant Metrewave Radio Telescope (uGMRT).

\section{Expectations from GMRT observations}

The Giant Metrewave Radio Telescope, and especially its upgraded version (uGMRT), constitutes undoubtedly the most powerful radio observatory in the $150 \mathrm{MHz}-1.4 \mathrm{GHz}$ range. This spectral domain is adequate to investigate non-thermal processes, and its use to investigate PACWBs comes as an obviousness. A description of the main specifications of the uGMRT are given by Ishwara-Chandra et al. (2019) in these proceedings.

At low radio frequencies, one has to remind that synchrotron spectra may undergo significant turnover. Let's comment on the relative efficiency of usual synchrotron turnover processes affecting astrophysical sources, emphasizing their potential relevance for the specific case of PACWBs:

- Synchrotron Self-Absorption (SSA). The self-absorption of synchrotron photons by the relativistic electron population responsible for their emission may become important in some circumstances, and strongly affect the synchrotron spectrum. Below a given turnover frequency, the optically thick spectrum is inverted, i. e. with a positive slope. The net effect is a significant drop of the synchrotron flux density at sufficiently low frequencies. The SSA process requires rather high number densities in relativistic electrons to significantly operate. Most recent predictions suggest that the SSA turnover should appear well below $100 \mathrm{MHz}$ in PACWBs, with potential exceptions for shorter period systems characterized by stronger magnetic fields in the emitting/self-absorbing region (De Becker 2018b). 
- The Razin-Tsytovitch effect. When a relativistic electron population is embedded in a thermal plasma, the relativistic beaming that drives the brightness of synchrotron emission can be suppressed, leading to a severe drop in the emission level. This plasma effect occurs below a turnover frequency that depends on the magnetic field strength and on the thermal electron number density. This effect may be active in PACWBs.

- Free-free absorption (FFA). The absorption of radio photons by thermal electrons in the plasma is the most likely turnover process that will severely affect the synchrotron spectrum. Depending on the plasma density and on geometrical thickness of the absorbing column, the FFA turnover may start to dominate close to $1 \mathrm{GHz}$, with more pronounced effects at lower frequencies. FFA is the main turnover process that will have to be taken into account to interpret radio spectra of PACWBs. Nice illustrations of this effect have for instance been presented by Dougherty et al. (2003), emphasizing the impact of FFA depending on the orientation and inclination of the system with respect to the line of sight.

The preparation of uGMRT observations of PACWBs can deeply benefit from the census of information already collected at $\mathrm{GHz}$ frequencies. Previous measurements significantly above $1 \mathrm{GHz}$ are very likely made in the optically thin part of the synchrotron spectrum. On the basis of flux densities in that domain, the spectral index can be measured (see blue vertical arrows in Fig. 4). The extrapolation of the spectrum at lower frequencies can be used to estimate flux densities at uGMRT bands. However, one should keep in mind that the occurrence of turnover processes as described above is expected to significantly reduce the measured flux densities. This extrapolation thus provides upper limits on the flux densities at uGMRT bands, that can significantly deviate from actual values.

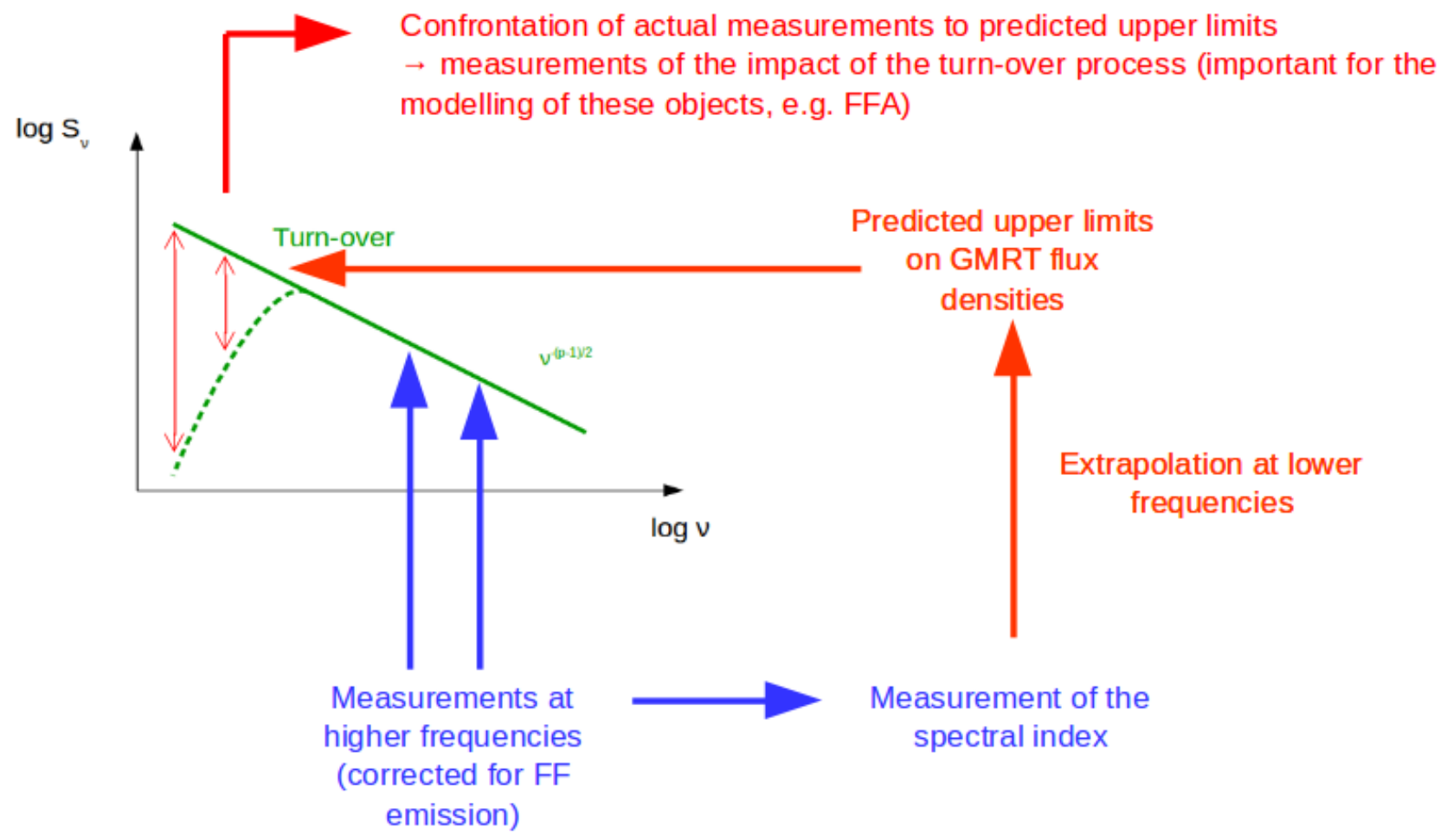

Figure 4: Illustration of the use of centimetric radio measurements (vertical blue arrows) to extrapolate flux densities at lower frequencies.

As a second step, actual measurements in that part of the spectrum can be made using the uGMRT. The confrontation of actual measurements to quantities extrapolated from the optically thin spectrum 
will provide a quantitative information on the impact of turnover processes in the low frequency part of the synchrotron spectrum. Optimally, this procedure should be repeated at several epochs to cover (a fraction of) the orbit of a given system. This would be especially useful to investigate the impact of FFA on the spectrum. As FFA is a strongly phase-locked process, the monitoring of its impact along the orbit would constitute a precious source of information for modelling purposes.

It is also very important to mention another relevant outcome of uGMRT observations: the identification of new PACWBs to be included in the catalogue. The extension of the current catalogue of PACWBs (De Becker \& Raucq 2013) is really important to address the issue of the fraction of particle accelerators among massive binaries (e.g. De Becker et al.2017). The organization of observation campaigns close to - and below $-1.4 \mathrm{GHz}$ is very likely to reveal additional non-thermal radio emitters, among which some may turn out to be massive binaries. For this purpose, the high sensitivity of uGMRT constitutes a considerable advantage. PACWBs are not very bright synchrotron radio emitters, as introduced in the energy budget discussion in Sect. 1 with the support of Fig. 2. To date, most PACWBs have been measured at radio frequencies at - or slightly below - the mJy level (exceptionally a few tens of mJy for the brightest ones). There is undoubtedly a wide field of discoveries to be made at the sub-mJy level, thus requiring the sensitivity of the uGMRT. This is especially true if one considers the impact of turnover processes (mainly FFA) that may substantially reduce the brightness of these objects at lower frequencies.

The variability of these sources must also be considered, as depending on the orbital phase the emission level may switch above and below the detection threshold. In order to improve the chance to achieve a relevant detection, repeated observations with sensitive radio instruments should be considered.

\section{Concluding remarks}

The investigation of Particle-Accelerating Colliding-Wind Binaries benefited of significant advances in the past decades. From the investigation of a few anomalies in radio spectra we switched to the investigation of a real class of objects. We now understand many aspects of their physics and we can use this understanding to make some predictions about members of this class in various spectral domains. However, our vision of this class of objects needs to be fed with scientific information, extracted in particular from observational measurements.

In this context, the upgraded Giant Metrewave Radio Telescope is clearly identified as a privileged tool:

- First of all, the spectral domain covered by the uGMRT is highly adequate to investigate nonthermal phenomena, notably thanks to a minimization of the contamination by thermal emission which can become significant above $1 \mathrm{GHz}$ (especially in binary configurations where the synchrotron emission is severely attenuated by free-free absorption).

- Second, the specific information that can be obtained for known PACWBs below $1 \mathrm{GHz}$ is important to measure and characterize in detail the impact of turnover processes. In particular, it is expected to help to identify the nature of the turnover process at work in the synchrotron spectrum, and a follow up of these processes as a function of the orbital phase will allow to study their dependence on physical parameters which characterize the colliding-wind system. The more detailed view of the low frequency spectrum of PACWBs offered by uGMRT observations will allow us to improve the modelling of the physics of these particle accelerators.

- Third, the unprecedented sensitivity of the uGMRT between $150 \mathrm{MHz}$ and $1.4 \mathrm{GHz}$ constitutes the key to measure synchrotron spectra for not so bright sources (down to a fraction of mJy), 
especially in a spectral domain significantly affected by turnover processes. In addition, such a sensitivity is a huge asset to search for additional members in the class of PACWBs. One can expect that a significant number of systems of this kind are still to be discovered. Such an endeavour is ambitious and requires the use of the most powerful tools.

Finally, it is worth to mention the strong connection between the radio processes discussed here, and prospects for high energy investigations. On the one hand, the combination of radio and high energy (hard X-rays and gamma-rays) will offer a more complete view of their non-thermal physics, and on the other hand present radio measurements can be used to constrain the properties of relativistic electron populations also likely to be involved in high energy non-thermal emission processes. The latter point may turn out to be important to make predictions and prepare future high energy observations. Here again next generation high energy observatories are expected to provide valuable information in the future to improve our understanding of the physics of PACWBs.

\section{Acknowledgements}

The authors would like to warmly thank the organizers for a fruitful and constructive workshop, along with BINA PIs and founders for pushing forward to make BINA such a promising initiative.

\section{References}

Abbott D. C., Bieging J. H., Churchwell E. 1984, ApJ, 280, 671

Abbott D. C., Beiging J. H., Churchwell E., Torres A. V. 1986, ApJ, 303, 239

Benaglia P., Romero G. E., Koribalski B., Pollock A. M. T. 2005, A\&A, 440, 743

Benaglia P., Koribalski B., Albacete Colombo J. F. 2006, PASA, 23, 50

Blomme R., Volpi D. 2014, A\&A, 561, A18

Blomme R., De Becker M., Runacres M. C. et al. 2007, A\&A, 464, 701

Blumenthal G. R., Gould R. J. 1970, Reviews of Modern Physics, 42, 237

De Becker M. 2018a, BSRSL, 87, 185

De Becker M. 2018b, A\&A, 620, A144

De Becker M., Raucq F. 2013, A\&A, 558, A28

De Becker M., Benaglia P., Romero G. E., Peri C. S. 2017, A\&A, 600, A47

Dougherty S. M., Pittard J. M., Kasian L. et al. 2003, A\&A, 409, 217

Dougherty S. M., Beasley A. J., Claussen M. J. et al. 2005, ApJ, 623, 447

Drury L. O'C. 1989, Rep. Prog. Phys., 46, 973

Dubner G., Giacani E. 2015, A\&ARv, 23, 3

Ensslin T. A., Biermann P. L., Klein U., Kohle S. 1998, A\&A, 332, 395

Ishwara-Chandra C. H., Benaglia P., De Becker M., Tej A. 2019, BSRSL, 88, 166 (this volume)

Montes G., Pérez-Torres M. A., Alberdi A., González R. F. 2009, ApJ, 705, 899

Panagia N., Felli M. 1975, A\&A, 39, 1

Puls J., Vink J. S., Najarro F. 2008, A\&ARv, 16, 209

Setia Gunawan D. Y. A., de Bruyn A. G., van der Hucht K. A., Williams P. W. 2000, A\&A, 356, 676

Setia Gunawan D. Y. A., de Bruyn A. G., van der Hucht K. A., Williams P. W. 2003, ApJS, 149, 123

Williams P. M., van der Hucht K. A., Pollock A. M. T. et al. 1990, MNRAS, 243, 662

Williams P. M., van der Hucht K. A., Spoelstra T. A. T. 1994, A\&A 291, 805

Wright A. E., Barlow M. J. 1975, MNRAS, 170, 41 